\title{
THE SETS THAT ARE SCISSOR CONGRUENT TO AN UNBOUNDED CONVEX SUBSET OF THE PLANE
}

BY

\author{
SYDELL PERLMUTTER GOLD
}

\begin{abstract}
It is shown that an unbounded convex plane body is scissor congruent to the union of a congruent body with a finite number of arbitrary topological discs. It is proved that 'is scissor congruent to' is an equivalence relation. Thus two unbounded convex plane bodies are scissor congruent if and only if the union of one with a finite number of topological discs is scissor congruent to the other.
\end{abstract}

1. Introduction. The decomposition of a set into a finite number of parts and the rearrangement of these parts to form another set has been studied in the past. Bolyai and Gerwin (see [4] for example) have shown that if two polygons have equal area one can be decomposed into a finite number of triangles which can be rearranged to form the other. Dubins, Hirsch and Karush showed [5] that if one cuts a strictly convex, compact, planar body, $E$, along arbitrary Jordan curves, the pieces can be rearranged by rigid motions to form another strictly convex planar body (i.e. the bodies are scissor congruent) if and only if the bodies have the same area and the boundary of one can be cut into a finite number of pieces which can be rearranged to form the boundary of the other (i.e. the boundaries are scissor congruent).

The notion of scissor congruence is herein expanded to unbounded bodies. An easily stated preliminary result is:

THEOREM 1.1. An unbounded convex body is scissor congruent to itself with a finite number of arbitrary topological discs adjoined.

This theorem implies, for example, that if a continuous function on the real line agrees with a convex function (e.g. a quadratic) except possibly on a compact interval, then the graph of the continuous function, together with its interior, is scissor congruent to the graph of the convex function together with its interior.

It will also be demonstrated that 'is scissor congruent to' is an equivalence relation and thus one can conclude:

Received by the editors May 28, 1974. 54D99.

AMS (MOS) subject classifications (1970). Primary 50B99, 52A10; Secondary 50A15,

Key words and phrases. Scissor congruent, unbounded figure, topological disc, topological half-plane, eventually convex, $S$-equivalent. 
THEOREM 1.2. Two unbounded convex bodies, $E_{+}$and $E_{-}$, are scissor congruent if and only if $E_{+}$together with a finite number of arbitrary topological discs is scissor congruent to $E_{-}$.

\section{Definition of scissor congruence.}

\section{DEFINITIONS.}

2.1. $\varnothing$ will denote the empty set.

2.2. A bounded $\operatorname{arc} A$ is the image of a connected subset $A^{\prime}$ of the circumference $C$ of a circle under a homeomorphism (of the plane onto itself) except that $\varnothing, C$ and one point sets are not considered as arcs. $A$ is open if $A^{\prime}$ is open in $C$.

2.3. $X+Y$ is the set with the same elements as $X \cup Y$, and is defined for sets whose interiors do not intersect. The set is called the sum of $X$ and $Y$.

2.4. Let $R$ be a rectangle of width $t>0$, and $A$ an open bounded arc contained in the interior of $R$ with the endpoints of $A$ contained in the opposite sides of length $t$ of $R$, with no endpoint of $A$ equal to a corner point of $R$. The two closed subsets, of sum $R$, defined by $A$ are each called a $W$-rectangle of width $t$. Two $W$-rectangles whose sum is a rectangle, $R$, are called a pair of $W$-rectangles of $R$.

2.5. A half-infinite strip, of width $t>0$, is a closed, unbounded, convex subset of the plane whose boundary is two parallel rays, a distance $t$ apart, and a line segment of length $t$ joining their endpoints.

LEMMA 2.6. A half-infinite strip is scissor congruent to the sum of a congruent strip and a W-rectangle of the same width.

Before proceeding with the proof, the relevant definitions will be given.

DEFINITIONS.

2.7. $\cong$ will denote congruence under plane rigid motion.

2.8. For any line segment $s,|s|$ will denote its length.

2.9. $d(\cdot, \cdot)$ will denote the Euclidean distance function. The appropriate domain will be clear from the context. (We will be working in Euclidean space throughout.)

2.10. For any set $S$, int $S$, ext $S$ and $\partial S$ will denote the interior, exterior and boundary of $S$, respectively.

2.11. A topological disc (topological half-plane) is the image of the closed unit disc (closed half-plane) under a homeomorphism of the plane onto itself.

Some notations, borrowed from [5], that will be used to define scissor congruence, are:

2.12. $D_{1}, \ldots, D_{m}, D_{-1}, \ldots, D_{-m}$ are $2 m$ topological half-planes; $D_{m+1}, \ldots, D_{n}, D_{-(m+1)}, \ldots, D_{-n}$ are $2(n-m)$ topological discs, $m \leqslant n<\infty$.

2.13. $T_{1}, \ldots, T_{n}$ are $n$ rigid motions of the plane. 
2.14. $E_{+}=\bigcup_{i>0} D_{i} ; E_{-}=\bigcup_{i<0} D_{i}$.

2.15. $K_{+}=\partial E_{+} ; K_{-}=\partial E_{-}$.

2.16. $K=K_{+} \cup K_{-}$.

2.17. $J_{i}=\partial D_{i}$, for all $i$.

2.18. $E_{+}$is scissor congruent to $E_{-}$if there is a collection of $D_{i}$ and $T_{i}$ which satisfy $2.12-2.14$ and if:

(i) $T_{i}\left(D_{i}\right)=D_{-i}, i>0$,

(ii) int $D_{i} \cap$ int $D_{j}=\varnothing$ for $i>j>0$ and for $i<j<0$.

The next assumption is automatic for unbounded figures:

(iii) $J_{i} \cap J_{j} \cap K_{+}$is a bounded set for $i>j>0 ; J_{i} \cap J_{j} \cap K_{-}$is a bounded set for $i<j<0$.

2.19. A collection $\left\{D_{i}, D_{-i}, T_{i}, i=1, \ldots, n\right.$, such that 2.18 (i)-(iii) is satisfied\} is a scissor congruence of $E_{+}$and $E_{-}$.

If two topological discs are contained in a union, all the residual domains of the union of their boundaries are topological discs (Kerékjártó's theorem; see [10], for example). Therefore, we can consider the sum, rather than the union, of a topological disc with a topological disc or topological half-plane in our proofs, without loss of generality.

For the remainder of this section we will assume that $t>0$.

ProOf OF LemMa 2.6. Let $R^{u}$ and $R^{b}$ be any pair of $W$-rectangles of a rectangle $R$. We can assume $R$ is contained in the upper half-plane with its lower boundary parallel to the $x$-axis. Let $T$ be the translation in the direction of the $+y$-axis so that $R \cap T(R)=$ a side of $R$. Let $U=R^{u}+T\left(R^{u}\right)+T^{2}\left(R^{u}\right)+$ $\cdots$ and $B=R^{b}+T\left(R^{b}\right)+T^{2}\left(R^{b}\right)+\cdots . U$ and $B$ are obviously topological half-planes and $E_{+}=U+B$ is a half-infinite strip and $E_{-}=R^{b}+T(U+B)$ is a $W$-rectangle + a half-infinite strip congruent to $E_{+}$.

Intuitively, $E_{+}$is scissor congruent to $E_{-}$because one can slip $U$ along the half-infinite strip and thus expose the $W$-rectangle, $R^{b}$. The slipped $U$, namely $T(U)$, is congruent to $U$, and $B$ is congruent to itself, i.e. $E_{+}$and $E_{-}$are scissor congruent as follows:

$$
\left.\right|_{(T(U)+B)=T(U+B)+R^{b}=E_{-} .} ^{U+B}
$$

LEMma 2.20. A half-infinite strip is scissor congruent to the union of a congruent strip and a topological disc.

For the proof of Lemma 2.20 we will need Lemma 2.22. 
Definition 2.21. If $p_{i}$ and $p_{j}$ are any two points of the plane, let $\overline{p_{i} p_{j}}$ denote the closed line segment with endpoints $p_{i}$ and $p_{j}$.

LEMmA 2.22. For any $t>0$ and any topological disc, $D$, there is $a$ (possibly empty) polygon, $P^{*}$, and a W-rectangle, $R^{*}$, of width $t$, such that $D+P^{*}$ is scissor congruent to $R^{*}$.

Before proceeding with the proof of Lemma 2.22 for the case of a general topological disc, it would be instructive to consider a convex topological disc, $D$. For any $t>0$, we can choose points $d_{1}, \ldots, d_{M}=d_{0} \in \partial D$ so that the sector $D_{i+1}$ bounded by $\overline{d_{i} d_{i+1}}$ and the subarc of $\partial D$ between $d_{i}$ and $d_{i+1}$ (call this subarc $A_{i+1}$ ) can be contained in the interior of a square of width $t$. Then, obviously, we can string the $D_{i}$ 's out so they are all contained in a rectangle, $R$, of width $t$. We can fill out $R$ with the polygon $D-\cup D_{i}$ and another polygon, $P^{*}$, if necessary, to obtain a $W$-rectangle, $R^{*}$.

The method for a general disc is more complicated, for we do not necessarily have sectors, but must define a finite number of small topological discs, $D_{i}$, with the property that $D-\cup D_{i}$ is a polygon.

Proof of Lemma 2.22. Let $S$ be the unit disc. By the Riemann Mapping Theorem there is a homeomorphism $f: S \rightarrow D$ which is a conformal map of int $S \rightarrow$ int $D$. Since $S$ is compact, $f$ is uniformly continuous. Therefore, given $t / 2>0$, there is a $\delta^{\prime}$ so that the closed disc, $S^{\prime}$, concentric with $S$ and of radius $1-\delta$, where $\delta=\min \left(1 / 2, \delta^{\prime}\right)$ is mapped into int $D$ and $\partial f\left(S^{\prime}\right)$ is within $t / 2$ of $\partial D$. $\partial f\left(S^{\prime}\right)$ is analytic and can therefore be approximated, within $t / 2$, by a polygonal line, $P \subset$ int $D$. Choose $M<\infty$ points, $d_{1}, \ldots, d_{M}=d_{0} \in \partial D$ so that $d\left(f^{-1}\left(d_{i-1}\right), f^{-1}\left(d_{i}\right)\right)<\delta, 1 \leqslant i \leqslant M$. For $i=1, \ldots, M$ : Let $p_{i}$ be a point of $P$ with $f^{-1}\left(p_{i}\right)$ on the radius to $f^{-1}\left(d_{i}\right) \in \partial S$ so that

$$
\overline{f^{-1}\left(p_{i}\right) f^{-1}\left(d_{i}\right)} \cap f^{-1}(P)=f^{-1}\left(p_{i}\right) \text {. }
$$

Let

$$
\begin{aligned}
U_{i}= & \overline{f\left(f^{-1}\left(p_{i}\right) f^{-1}\left(d_{i}\right)\right)}, \\
A_{i}= & \text { the closed subarc of } \partial D \text { with end points } d_{i-1} \\
& \text { and } d_{i} \text {. Without loss of generality we can } \\
& \text { assume int } \left.A_{i} \cap \operatorname{int} A_{j}=\varnothing \text { for } i \neq j .\right) \\
L_{i}= & \text { the closed polygonal line segment of } P \text { joining } p_{i-1} \text { and } p_{i} .
\end{aligned}
$$

Then the closed set, $D_{i}$, bounded by $U_{i-1}+A_{i}+U_{i}+L_{i}$ is a topological disc since it is the homeomorphic image of a topological disc contained in $S$. Choose $T_{i}$ to be a rigid motion satisfying the following conditions:

(i) $T_{i}\left(\overline{d_{i-1} d_{i}}\right)$ are consecutive segments along some oriented line, $L$, with nonintersecting interiors. $T_{i}\left(\overline{d_{i-1} d_{i}}\right)$ is before $T_{j}\left(\overline{d_{j-1} d_{j}}\right)$ for $i<j$. 
(ii) int $D_{i} \cap$ int $D_{j}=\varnothing$, for $i<j<0$, where $T_{i}\left(D_{i}\right)=D_{-i}$.

(iii) The polygonal arcs, $T_{i}\left(L_{i}\right)$ are all on the same side of $L$.

(iv) $d\left(T_{1}\left(d_{0}\right), T_{M}\left(d_{M}\right)\right)<\infty$.

Thus the $D_{-i}$ 's will lie within a rectangle, $R$, of width $t$. Join $T_{1}\left(p_{0}\right)$ and $T_{M}\left(p_{M}\right)$ to the nearest side (but not a corner) of $R$ by a line segment. Join $T_{j}\left(p_{j}\right)$ to $T_{j+1}\left(p_{j}\right)$ for $j=1, \ldots, M-1$, by a polygonal line, $L^{\prime}$, which intersects none of the $D_{-i}$ 's so that $A=\bigcup_{i} T_{i}\left(U_{i-1} \cup A_{i} \cup U_{i}\right) \cup L^{\prime}$ is an arc. We have thus defined a $W$-rectangle, $R^{b} \subset R$, whose nonlinear boundary segment is $A$. Obviously $\bigcup_{i} D_{-i} \subset R^{b}$. Let $P^{b}=R^{b}-\bigcup_{i<0} D_{i}$. $P^{b}$ is a finite union of polygons, and $\bar{P}=D-\bigcup_{i>0} D_{i}$ is a finite union of polygons.

If area $\bar{P} \leqslant$ area $P^{b}$ then let $P^{*}$ be any polygon disjoint from $D$ with area $P^{*}=$ area $P^{b}-$ area $\bar{P}$. Then $\bar{P}+P^{*}$ is scissor congruent to $P^{b}$ by Bolyai's theorem [4] and hence $D+P^{*}$ is scissor congruent to $R^{b}\left(=R^{*}\right)$, a $W$-rectangle.

If area $P>$ area $P^{b}$, then $\bar{P}$ is scissor congruent to $P^{b}+$ a rectangle of width $t$ adjoined to $R^{b}$ and sharing a side with $R^{b}$, i.e. another $W$-rectangle, $R^{*}$, of width $t$ and $P^{*}$ is empty.

Proof of Lemma 2.20. Without loss of generality, we can assume the strip and the topological disc have disjoint interior. Then this lemma follows from 2.22 and 2.6 since we do not decompose the $W$-rectangle, $R^{b}$, in Lemma 2.6 to obtain scissor congruence. That is: Let $I$ and $I^{\prime}$ be half-infinite strips of width $t$. Let $D$, $P^{*}$ and $R^{*}$ be as in Lemma 2.22 . We can choose $P^{*}$ to be a rectangle so that $I+P^{*} \cong I$. Then $I+P^{*}+D$ is scissor congruent to $I^{\prime}+R^{*}$. But we can slip $R^{*}$ into $I^{\prime}$ as in Lemma 2.6 and thus have $I+P^{*}+D$ scissor congruent to $I^{\prime}$.

LEMMA 2.23. A half-infinite strip of width $t$ is scissor congruent to the union of a congruent strip with a finite number of topological discs.

Proof. Follows from Lemma 2.20 if we note that we can sum the $W$-rectangles associated with each topological disc to form another $W$-rectangle.

DEFinItion 2.24. A strip of width $t$ is the union of a half-infinite strip of width $t$ with a finite number of topological discs.

LEMma 2.25. Two strips of the same width are scissor congruent.

Proof. Follows immediately from Lemma 2.23 , noting that if the strips are of width $t$, the topological discs of each can be made scissor congruent to a $W$-rectangle of width $t / 2$.

\section{Definition of $S$-equivalence.}

DEFINITIONS.

3.1. A bounded figure is the union of a finite number of topological discs. An unbounded figure is the union of a topological half-plane and a bounded figure.

3.2. An unbounded figure is eventually convex if it is a union of an 
unbounded convex figure and a finite number of topological discs.

3.3. An axis of a set $E$ is a ray which is a subset of $E$. Grünbaum, on p. 23 of [6], proves two lemmas which can be stated as follows:

LEMMA 3.4. Every unbounded convex figure has at least one axis.

LEMMA 3.5. If $X$ is an axis of a convex figure, $E$, and if $T$ is a translation with $T$ (endpoint of $X$ ) $\in E$, then $T(X)$ is an axis of $E$.

If $E$ is an unbounded convex figure in the plane, we will assume, without loss of generality, that:

(i) $E$ is contained in the closed upper half-plane,

(ii) the $x$-axis is a line of support of $E$, and

(iii) $E$ has an axis perpendicular to the $x$-axis, say $x=X$.

Let $g$ be a convex function so that the boundary of $E$ is the graph of $g$. Then, if $x_{1}$ and $x_{2}$ are any two real numbers, let

$$
G\left(x_{1}, x_{2}\right)=x_{2} /\left(g\left(x_{1}+x_{2}\right)-g\left(x_{1}\right)\right) \text {. }
$$

$G\left(x_{1}, x_{2}\right)$ is a monotone function in $x_{2}$ for a fixed $x_{1}$ since $g$ is convex. Therefore, the following limits exist:

$$
\inf _{x_{1} \geqslant X} \sup _{x_{2} \downarrow 0} G\left(x_{1}, x_{2}\right)=\inf _{x_{1} \geqslant X}\left(g_{R}^{\prime}\left(x_{1}\right)\right)^{-1}=\tan B_{R}(E)
$$

and

$$
\inf _{x_{1} \leqslant X} \sup _{x_{2} \uparrow 0} G\left(x_{1}, x_{2}\right)=\inf _{x_{1} \leqslant X}\left(g_{L}^{\prime}\left(x_{1}\right)\right)^{-1}=\tan B_{L}(E)
$$

where $g_{R}^{\prime}\left(x_{1}\right)$ and $g_{L}^{\prime}\left(x_{1}\right)$ are the right-hand and left-hand derivatives of $g$ at $x_{1}$, respectively.

DEFINITIONS.

3.6. If $E$ is situated in the plane so that (i)-(iii) above hold, and also so that $\left|B_{R}(E)\right|=\left|B_{L}(E)\right|$, we will say that $E$ is properly situated.

3.7. The angle of $E$ is $\left|B_{R}(E)\right|+\left|B_{L}(E)\right|$, the maximum angle between axes of $E$.

3.8. $E$ is uniaxial if the angle of $E$ is 0 , and multiaxial otherwise. For example, a figure whose boundary is the graph of a parabola is uniaxial. One whose boundary is the graph of one branch of a hyperbola is multiaxial.

Note that all the axes of a uniaxial figure are translates of each other. Without loss of generality, we will consider all unbounded convex figures to be properly situated in the plane.

DeFinitions.

3.9. An unbounded arc is the image of a half-infinite interval under a homeomorphism of the plane onto itself. It is open or closed according to whether the 
interval is open or closed. The word arc will refer to bounded or unbounded arcs.

3.10. Let $V_{+}$and $V_{-}$be eventually convex figures.

(i) $\partial V_{+}$is eventually scissor congruent to $\partial V_{-}$if and only if there exist four unbounded open arcs, $C_{1}, C_{2} \in \partial V_{+}$which are disjoint, and $C_{-1}, C_{-2} \in \partial V_{-}$ which are also disjoint, and there exist rigid motions $M_{1}$ and $M_{2}$ such that $M_{i}$ : $C_{i} \cong C_{-i}, i=1,2$.

(ii) $V_{+}$is $S$-equivalent to $V_{-}$if and only if:

(a) $\partial V_{+}$is eventually scissor congruent to $\partial V_{-}$, with $M_{1}$ and $M_{2}$ translations. Furthermore, if $V_{+}$and $V_{-}$are uniaxial, we require that

(b) $M_{1}(N)=M_{2}(N)$, where $N$ is a line parailel to an axis of $V_{+}$.

We will show in Theorem 9.1 that for two eventually convex figures to be scissor congruent it is necessary and sufficient that they be $S$ equivalent.

LEMMA 3.11. S-equivalence is an equivalence relation on the class of eventually convex figures.

Before proceeding with the proof, we will demonstrate the following lemmas.

LEMMA 3.12. 'Is eventually scissor congruent to' is a transitive relation on the class of boundaries of unbounded figures.

Proof. Let $E_{+}, E_{-}, E_{*}$ be unbounded figures with boundaries $K_{+}, K_{-}$, $K_{*}$ respectively. Assume that $K_{+}$is eventually scissor congruent to $K_{-}$and $K_{-}$ is eventually scissor congruent to $K_{*}$. For $i=1,2$ : There are four unbounded $\operatorname{arcs} A_{i} \subset K_{+}, A_{-i} \subset K_{-}$with $R_{i}: A_{i} \cong A_{-i}, R_{i}$ rigid motions, and $A_{1} \cap A_{2}=$ $A_{-1} \cap A_{-2}=\varnothing$. Similarly, there are four unbounded $\operatorname{arcs} A_{-i}^{\prime} \subset K_{-}, A_{* i}^{\prime} \subset K_{*}$ with $R_{i}^{\prime}: A_{-i}^{\prime} \cong A_{* i}^{\prime}, R_{i}^{\prime}$ rigid motions, and $A_{-1}^{\prime} \cap A_{-2}^{\prime}=A_{* 1}^{\prime} \cap A_{* 2}^{\prime}=\varnothing$.

Let us assume the arcs are indexed so that either $A_{-i} \subset A_{-i}^{\prime}$ or else $A_{-i}^{\prime} \subset$ $A_{-i}$. Let $T^{i}=R_{i}^{\prime} R_{i}, B_{i}=\left(T^{i}\right)^{-1} A_{* i}^{\prime}$. If $B_{i} \subset A_{i}$, let $U_{i}=B_{i}$. If $B_{i} \supset A_{i}$, let $U_{i}=A_{i}$. Let $U_{* i}=T^{i}\left(U_{i}\right)$.

We have thus defined four unbounded arcs, $U_{1}, U_{2}$ of $K_{+}$which are disjoint, and $U_{* 1}, U_{* 2}$ of $K_{*}$ which are disjoint with $T^{i}: U_{i} \cong U_{* i}$. Thus $K_{+}$is eventually scissor congruent to $K_{*}$.

LEMMA 3.13. If two unbounded convex figures are S-equivalent they have equal angles.

Proof. Using the notation of 3.10, suppose $L$ is an axis of $V_{+}$with endpoint the same as that of $C_{1}$, say $c_{1}$. Obviously, $M_{1}(L) \cap C_{-1}=M_{1}\left(c_{1}\right)$. Suppose $M_{1}(L) \cap C_{-2} \neq \varnothing$, say equals $c_{-2}$. Let $L_{-}=M_{1}(L) \cap$ ext $V_{-}$. Then $M_{2}^{-1}\left(L_{-}\right) \in \operatorname{ext} V_{+}$. But $M_{2}^{-1}\left(L_{-}\right)$has endpoint belonging to $C_{2}$ and, since $M_{1}$ and $M_{2}$ are translations, by Lemma 3.5 is an axis of $V_{+}$. Contradiction.

Therefore $M_{1}(L) \cap C_{-2}=\varnothing$, i.e. $M_{1}(L)$ is an axis of $V_{-}$. Similarly we can 
show that $M_{1}^{-1}$ of any axis of $V_{-}$is also an axis of $V_{+}$. It follows from Lemma 3.5 that the angles of $V_{+}$and $V_{-}$are equal.

Proof of Lemma 3.11. S-equivalence is obviously a reflexive and symmetric relation. Using the notation of Lemma 3.12, assume that $E_{+}$is $S$ equivalent to $E_{-}$and $E_{-}$is $S$-equivalent to $E_{*}$.

Then by Lemma $3.12, K_{+}$is eventually scissor congruent to $K_{*}$.

$T^{1}$ and $T^{2}$ are translations since $R_{1}, R_{2}, R_{1}^{\prime}$ and $R_{2}^{\prime}$ are. If $E_{+}$is uniaxial then so are $E_{-}$and $E_{*}$ by Lemma 3.13 . If $L$ is a line parallel to an axis of $E_{+}$, then

$$
T^{1}(L)=R_{1}^{\prime} R_{1}(L)=R_{1}^{\prime} R_{2}(L)=R_{2}^{\prime} R_{2}(L)=T^{2}(L) .
$$

Therefore $E_{+}$is $S$-equivalent to $E_{*}$.

4. Sequivalence implies scissor congruence.

DEFINITIONS.

4.1. A special wedge, $E$, is an unbounded convex figure bounded by two rays with the same endpoint, $x$. The angle, $\theta$, between the rays is the angle of $E$. $0<\theta \leqslant \pi . x$ is the apex of $E$. The axis of $E$ with endpoint $x$ which bisects $\theta$ is called the center line of $E$.

4.2 Let $E$ be a special wedge and $P$ a closed half-plane so that $E-E \cap P$ is bounded. Let $D$ be a bounded figure with int $D \cap$ int $P=\varnothing$. Then $W=D+$ $E \cap P$ is a wedge. Obviously, the angle of $W$ is the angle of $E .(D, E, P)$ will denote the triple associated with $W$.

LEMMA 4.3. If two wedges have equal angles they are scissor congruent.

Proof. Let $W_{+}$and $W_{-}$be two wedges with equal angles. Let $\left(D_{+}, E_{+}, P_{+}\right)$ and $\left(D_{-}, E_{-}, P_{-}\right)$be the triples associated with $W_{+}$and $W_{-}$, respectively.

Case (a). Angle of $E_{+}=$angle of $E_{-} \neq \pi . E_{+}$and $E_{-}$are congruent special wedges which we can assume are properly situated. Therefore there is a closed upper half-plane, $P$, such that:

(i) $H_{+}=E_{+} \cap P_{+} \cap P \cong E_{-} \cap P_{-} \cap P=H_{-} \neq \varnothing$ and

(ii) $E_{+}-H_{+}$and $E_{-}-H_{-}$are bounded and nonempty.

Let $x_{i}\left(x_{-i}\right)$ be the endpoint of the ray $X_{i}\left(X_{-i}\right)$ of the boundary of $H_{+}\left(H_{-}\right)$, for $i=1,2$. Let $Y_{1}\left(Y_{-1}\right)$ be an axis of $H_{+}\left(H_{-}\right)$parallel to $X_{2}\left(X_{-2}\right)$ with endpoint $x_{1}\left(x_{-1}\right)$. The special wedge, $E_{+}^{\prime}$, bounded by $X_{1}$ and $Y_{1}$ is congruent to the special wedge, $E_{-}^{\prime}$, bounded by $X_{-1}$ and $Y_{-1}$.

Let $I_{+}=$the strip $\left(H_{+}-E_{+}^{\prime}\right)$ and $I_{-}=$the strip $\left(H_{-}-E_{-}^{\prime}\right) . I_{+}$and $I_{-}$ are congruent strips, so the strip $I_{+}+\left(W_{+}-H_{+}\right)$is scissor congruent to the strip $I_{-}+\left(W_{-}-H_{-}\right)$, by Lemma 2.25 .

Case (b). If $E_{+}$and $E_{-}$are closed half-planes, let $I_{+} \subset E_{+}$and $I_{-} \subset E_{-}$ be congruent half-infinite strips whose boundary rays are perpendicular to the 
boundary of $E_{+}$and $E_{-}$, respectively. Then $I_{+}+D_{+}$and $I_{-}+D_{-}$are scissor congruent strips and $E_{+}-I_{+}$and $E_{-}-I_{-}$are congruent.

In either case, $W_{+}$is scissor congruent to $W_{-}$.

THEOREM 4.4. If two eventually convex figures are S-equivalent they are scissor congruent.

Proof. We will use the notation of Definition 3.10. For $i=1,2$ : Let $c_{i}$, $c_{-i}$ be the endpoint of $C_{i}$ and $C_{-i}$, respectively. We can assume $c_{1} \neq c_{2}$ and $c_{-1} \neq$ $c_{-2}$. Let $R_{i}$ be an axis of $V_{+}$with endpoint $c_{i}$, and for $V_{+}$multiaxial we require that $R_{1}$ and $R_{2}$ not be parallel or collinear and $R_{1} \cap R_{2}=\varnothing$.

Let $R_{-i}=M_{i}\left(R_{i}\right)$. Then $D_{i}$, with boundary $C_{i}+R_{i}$ and $D_{-i}$, with boundary $C_{-i}+R_{-i}$ are (possibly empty) topological half-planes and $D_{-i}=M_{i}\left(D_{i}\right)$. We have defined $D_{i}$ and $D_{-i}$ so that int $D_{1} \cap$ int $D_{2}=$ int $D_{-1} \cap$ int $D_{-2}=\varnothing$. Thus $D_{1}+D_{2}$ is scissor congruent to $D_{-1}+D_{-2}$.

$V_{+}-\left(D_{1}+D_{2}\right)$ and $V_{-}-\left(D_{-1}+D_{-2}\right)$ are:

(i) wedges, if $V_{+}$and $V_{-}$are multiaxial. They have the same angle since $M_{1}$ and $M_{2}$ are translations, and are therefore scissor congruent by Lemma 4.3.

(ii) They are strips of the same width if $V_{+}$and $V_{-}$are uniaxial, since by 3.10 (ii)(b)

$$
d\left(R_{-1}, R_{-2}\right)=d\left(M_{1}\left(R_{1}\right), M_{2}\left(R_{2}\right)\right)=d\left(M_{2}\left(R_{1}\right), M_{2}\left(R_{2}\right)\right)=d\left(R_{1}, R_{2}\right) .
$$

Thus they are scissor congruent by Lemma 2.25. For either case $V_{+}$and $V_{-}$are scissor congruent.

Proof of THEOREM 1.1. An unbounded convex figure is obviously $S$-equivalent to the union of a congruent figure with a bounded figure.

Thus this theorem follows from Theorem 4.4.

\section{Wedges.}

LEMMA 5.1. Two special wedges which are scissor congruent have equal angles.

We will establish some lemmas needed for the proof of this proposition.

Definition 5.2. A section, $Z$, of a special wedge, $E$, is a trapezoid contained in $E$ such that:

(i) the parallel sides of $Z$, called the base and upper boundary, are perpendicular to the center line of $E$, with |base $\mid$ |upper boundary|.

(ii) The nonparallel sides are contained in the boundary of $E$.

Let $E_{+}$and $E_{-}$be scissor congruent special wedges. Using the notation of Definition 2.18, we can state the following.

Definition 5.3. Two sections, $z_{+}$of $E_{+}$and $z_{-}$of $E_{-}$, are $a$ pair of sections if and only if: 
(i) the closed half-plane containing $z_{+}$with boundary containing the base line of $z_{+}$, contains no topological discs of the scissor congruence of $E_{+}$and $E_{-}$.

(ii) $S_{-}=\bigcup_{1 \leqslant i \leqslant m} T_{i}\left(D_{i} \cap z_{+}\right)$contains $z_{-}$.

\section{LEMMA 5.4. There exists a pair of sections.}

Proof. Let $z_{+}$be a section satisfying 5.3(i). Such a section exists since there are only a finite number of topological discs in a scissor congruence. Let $b_{+}$ be the base and $b_{+}^{\prime}$ the upper boundary of $z_{+}$. Intuitively, if the endpoints of $T_{i}\left(b_{+}\right)$for each $i$ are closer to the apex of $E_{-}$than the endpoints of $T_{i}\left(b_{+}^{\prime}\right)$ for each $i$, then there are certainly sections in $S_{-}$. These notions will now be described more rigorously. Let $x$ be the apex of $E_{-}, X$ its center line. Define $\Pi: E_{-} \rightarrow X$ to be the perpendicular projection of $E_{-}$onto $X$. Let $b$ be any line segment in $E_{+}$perpendicular to its center line.

For $1 \leqslant i \leqslant m$ with $b \cap D_{i} \neq \varnothing$ : Let $c_{i}(b)$ and $f_{i}(b)$ be the endpoints of $\Pi\left(T_{i}(b) \cap E_{-}\right)$where $d\left(c_{i}(b), x\right) \leqslant d\left(f_{i}(b), x\right)$. Let

$$
\begin{gathered}
c(b)=\underset{i}{\operatorname{minimum}} d\left(c_{i}(b), x\right), \\
f(b)=\underset{i}{\operatorname{maximum}} d\left(f_{i}(b), x\right) .
\end{gathered}
$$

If $f\left(b_{+}\right)<c\left(b_{+}^{\prime}\right)$ then $S_{-}$will contain at least one section. Since $E_{+}$is unbounded we can always find a $b_{+}^{\prime}$ parallel to $b_{+}$satisfying this inequality. Let $z_{-} \subset S_{-}$ have base $b_{-}$and upper boundary $b_{-}^{\prime}$ where $d\left(b_{-}, x\right)=f\left(b_{+}\right)$and $d\left(b_{-}^{\prime}, x\right)=$ $c\left(b_{+}^{\prime}\right)$, with the sides of $z_{-} \subset \partial E_{-}$. Thus $z_{-}$is a section of $E_{-}$. Thus $z_{+}$and $z_{-}$ satisfying 5.3 (i) and (ii) have been constructed and so are a pair of sections.

DEFINITION 5.5. Using the notation of 5.3 and 5.4, a pair of sections $z_{+}$ and $z_{-}$is allowable if and only if 5.3 (i) and (ii) are satisfied and $d\left(b_{-}, x\right)=f\left(b_{+}\right)$ and $d\left(b_{-}^{\prime}, x\right)=c\left(b_{+}^{\prime}\right)$. Obviously there is an allowable pair of sections, $Z_{+}$and $Z_{-}$, with base $b_{+}$and $b_{-}$, respectively, and with $z_{+} \subset Z_{+}$and $z_{-} \subset Z_{-}$, having height as large as we wish.

Using the notation we have just developed, we will proceed with the proof of Lemma 5.1 .

Proof of Lemma 5.1. Let $2 \theta_{+}$be the angle of $E_{+}, 2 \theta_{-}$that of $E_{-}$, with $0<\theta_{+}<\pi / 2 ; 0<\theta_{-} \leqslant \pi / 2$. Let $W=\left|b_{+}\right|, W^{\prime}=\left|b_{-}\right| . W^{\prime}$ is determined by our choice of $W$ and the scissor congruence. Let $l_{+}, l_{-}, L_{+}$and $L_{-}$be the height of the sections $z_{+}, z_{-}, Z_{+}$and $Z_{-}$, respectively. Then, if we let $L_{+}-l_{+}=\gamma$ and $l_{+}-l_{-}=\mu$, with $\mu \geqslant 0, L_{+}-L_{-}=\gamma\left(1-\cos \theta_{-}\right)+\mu$. Let $N=\gamma\left(1-\cos \theta_{-}\right)$ $+\mu$. Since area $Z_{+} \geqslant$area $Z_{-}$we have

$$
W L_{+}+L_{+}^{2} \tan \theta_{+} \geqslant W^{\prime}\left(L_{+}-N\right)+\left(L_{+}-N\right)^{2} \tan \theta_{-} .
$$

Therefore, 


$$
W^{\prime} N-N^{2} \tan \theta_{-} \geqslant L_{+}^{2}\left(\tan \theta_{-}-\tan \theta_{+}\right)+N^{\prime} L_{+}
$$

where $N^{\prime}=W^{\prime}-2 N \tan \theta_{-}-W$. Completing the square and taking square roots we obtain

$$
\begin{aligned}
\left\{W^{\prime} N\right. & \left.-N^{2} \tan \theta_{-}+\frac{N^{\prime 2}}{4\left(\tan \theta_{-}-\tan \theta_{+}\right)}\right\}^{1 / 2} \\
& -\frac{N^{\prime}}{2\left(\tan \theta_{-}-\tan \theta_{+}\right)^{1 / 2}} \geqslant L_{+}\left(\tan \theta_{-}-\tan \theta_{+}\right)^{1 / 2} .
\end{aligned}
$$

This contradicts the unboundedness of $L_{+}$unless $\tan \theta_{-}=\tan \theta_{+}$, i.e. $\theta_{-}=\theta_{+}$.

LEMMA 5.6. If two wedges are scissor congruent their angles are equal. (The converse of Lemma 4.3.)

Proof. Let $W_{+}$and $W_{-}$be scissor congruent wedges with associated triples $\left(D_{+}, E_{+}, P_{+}\right)$and $\left(D_{-}, E_{-}, P_{-}\right)$, respectively. Then, without loss of generality, we can make the additional requirement that $z_{+}$and $z_{-}$lie in $E_{+} \cap P_{+}$and $E_{-} \cap P_{-}$, respectively, in the proof of Lemma 5.1. Then the proof of Lemma 5.1 is also valid for wedges.

6. The boundaries $K_{+}$and $K_{-}$. In this and the following two sections, the assumption that $E_{+}$is scissor congruent to $E_{-}$is implicit. We choose a particular scissor congruence and use the notation of Definition 2.18.

The definition of scissor congruence in [5] only allows the intersection of 2.18 (iii) to be finite, rather than bounded. The first six lemmas proved in [5] are based on properties of topological discs that are also properties of topological halfplanes. They will be labeled Lemma 1 [5], etc. to show that they are taken from [5]. The lemmas that do not use assumption 2.18(iii) will be stated without proof.

LEMma 1 [5]. For all $i,\left(J_{i}-\bigcup_{j \neq i} J_{j}\right) \subset K$.

DEFINITION [5].

$$
V=\bigcup_{i \neq j \neq k ; i \neq k}\left(J_{i} \cap J_{j} \cap J_{k}\right) \cup \bigcup_{i \neq j}\left(J_{i} \cap J_{j} \cap K\right) .
$$

Using (ii) and (iii) of 2.18 , it can be easily seen that $V$ is a bounded set.

DEFinition [5]. For $i>0, T_{-i}=T_{i}^{-1}$. Let us introduce two transformations $T$ and $R$ defined for certain ordered couples $(x, i)$. For $x \in J_{i}$, let $T(x, i)=$ $\left(T_{i} x,-i\right)$. For $x \in J_{i} \cap J_{j}-V$, with $i \neq j$, let $R(x, i)=(x, j)$. Since $x \notin V, j$ is unique, so $R$ is well defined.

For any function $f$, let $D(f)$ denote its domain and $R(f)$ its range. Let $1_{X}$ denote the identity map of a set $X$ onto itself.

Lemma 2 [5]. Let $A$ be an arc, $A \subset\left(J_{i}-V\right)$. Then $A \subset K$ or $A \subset J_{j}$ for some $j \neq i$. 
Lemma 3 [5]. (a) $D(R)=\left\{(x, i): x \in J_{i}\right.$ and $\left.x \notin K \cup V\right\}$.

(b) $R(R)=D(R)$, and $R^{2}=1_{D(R)}$.

(c) $R(T)=D(T)$, and $T^{2}=1_{D(T)}$. $k \geqslant 0$.

(d) $R\left((T R)^{k} T\right)=D\left((T R)^{k} T\right)$, and $\left((T R)^{k} T\right)^{2}=1_{D\left((T R)^{k} T\right)}$, for each

Lemma 4 [5]. Assume (a) $x, y \in K$, (b) $(x, i) \in D\left((T R)^{k} T\right)$, and (c) $(y, j) \in D\left((T R)^{h} T\right)$.

Then $(T R)^{k} T(x, i)=(T R)^{h} T(y, j)$ only if $k=h$ and $(x, i)=(y, j)$.

Definition [5]. $W=\left\{x: x \in K\right.$ and there exist $i, k, v, i^{\prime}$ such that $v \in V$, $(x, i) \in D\left((T R)^{k} T\right)$ and $\left.(T R)^{k} T(x, i)=\left(v, i^{\prime}\right)\right\}$.

Lemma 5 [5]. $W$ is bounded.

Proof. For every $x \in W$, the set of all $\left(v, i^{\prime}\right)$ such that for some $(i, k)$ $\left(i, k, v, i^{\prime}\right)$ satisfies the definition is nonempty. Distinct $x$ 's correspond to disjoint sets by Lemma 4 [5]. $V \cap W$ is bounded since $V$ is bounded. $(W-V) \cap \bigcup_{i>m} J_{i}$ is bounded since each such $J_{i}$ is bounded. Suppose $x \in(W-V) \cap \bigcup_{i \leqslant m} J_{i}$. It follows from 2.18 (iii) that for all but a bounded set of such $x$ 's, $x \in J_{i} \cap W$ for exactly one $i$. Therefore, for each such $x$ there is exactly one pair $(x, i)$ and hence exactly one $\left(i, k, v, i^{\prime}\right)$ satisfying the definition of $W$. (If there were another, say (i,h, $\left.v^{\prime}, j^{\prime}\right)$, with $k<h$, then $v \in D(R)$ which is impossible.) But for all but a bounded set of $x$ 's, if $x \in J_{i} \cap W$, there is a $k=k^{\prime}$ such that $(x, i) \in D\left((T R)^{k^{\prime}} T\right)$. Thus, except for a bounded set of $x$ 's, there are only a finite number of rigid motions mapping the $(x, i)$ pairs onto the $\left(v, i^{\prime}\right)$ pairs. Since $V$ is bounded and there are only a finite number of values of $i$ and $i^{\prime}, W$ must be bounded.

LEMMA 6 [5]. $K-(V \cup W)$ is a union of disjoint open arcs, each included in some $J_{i}$, and this decomposition is unique. Moreover, this decomposition contains a finite number of unbounded arcs.

Proof. The proof of the first part of this lemma follows that of Lemma 6 in [5]. For the last part note that $K \subset \bigcup_{i} J_{i}$, so $K-(V \cup W)=\bigcup_{i}\left(K \cup\left(J_{i}-(V \cup W)\right)\right)$. $V \cup W$ is bounded, so $J_{i}-(V \cup W)$ is uniquely expressible as a union of disjoint open arcs. If $D_{i}$ is a topological half-plane, this union contains at most two unbounded open arcs of $J_{i}$ and thus only a finite number of unbounded arcs.

Definition [5]. $p(x)=x^{\prime}$ if $x, x^{\prime} \in K-(V \cup W)$ and there exist $i, i^{\prime}$ and $k$ such that $(x, i) \in \mathcal{D}\left((T R)^{k} T\right)$ and $(T R)^{k} T(x, i)=\left(x^{\prime}, i^{\prime}\right)$.

It is shown in [5] that $p$ is well defined and $p^{2}=1 D(p)$.

Definition [5]. Let $A$ be the set of open arcs described in Lemma 6 [5].

It is proved in Lemma 7 [5] that, for bounded figures, the domain of $p$ is all of $K-(V \cup W)$. This is not necessarily true for unbounded figures even if we. 
restrict $V$ and $W$, and thus $A$, to be finite sets.

Intuitively, some arcs of $A$ are mapped onto the boundary, $K$, by $(T R)^{r} T$ for some integer $r$. The other arcs of $A$ are mapped into the interior of $E_{+}$or $E_{-}$by $T$ and their images under $T$ are mapped out of the interior by no $(T R)^{r}$. The former belong to the domain of $p$, and the latter do not. For $C$ a bounded open arc of $A$ with $C \not \subset D(p)$, there exists an $i$ such that $(T R)^{r} T\left(C, i_{r}\right) \subset J_{i}$ for infinitely many values of $r$, say $r_{1}, r_{2}, \ldots$, and $(T R)^{r_{s}} T\left(C, i_{r_{s}}\right) \cap(T R)^{r_{j}} T\left(C, i_{r_{j}}\right)=$, with $s \neq j$. I.e. $J_{i}$ contains infinitely many disjoint open subarcs each of which is congruent to $C$.

The goal now is to show that the unbounded arcs of $K-(V \cup W)$ are in the domain of $p$. Let us now restrict $E_{+}$and $E_{-}$to be unbounded figures. If the set $V$ is empty, then $E_{+}$and $E_{-}$are congruent figures and we need proceed no further. So we can assume $V \cup W$ has at least one element which belongs to $K_{+}$, and consequently another element which belongs to $K_{-}$. Therefore, there are precisely four arcs $A^{1}, A^{2}, A^{-1}, A^{-2} \in A$ which are unbounded.

Definition 6.1. Let $U=\left\{A^{1}, A^{2}, A^{-1}, A^{-2}\right\}$.

Lemma 6.2. If $A \in U$ then $A \subset D(p)$. Moreover, there exist $i_{0}, i_{1}, \ldots, i_{k}$ such that for $0 \leqslant r \leqslant k$ : (a) $-i_{r} \neq i_{r+1}$; (b) $A \subset J_{i_{0}}$; and, letting $A_{r}$ denote $T_{i_{r}} \cdots T_{i_{0}} A$, (c) $A_{r} \subset J_{-i_{r}} \cap J_{i_{r+1}}$; (d) $p(A)=A_{k} \subset J_{-i_{k}} \cap(K-(V \cup W))$.

Proof. Fix $A \in U$. Then $A$ is a subarc of some unique $J_{i}$, say $i=i_{0}$. Let $(A, i)$ be the set of all $(x, i)$ for which $x \in A$. Then $\left(A, i_{0}\right) \subset D(T)$ and $T\left(A, i_{0}\right)$ $=\left(A_{0},-i_{0}\right)$, where $A_{0}=T_{i_{0}} A$ is congruent to $A$. It is shown in Lemma 7 [5] that: if $\left(r, A_{r},-i_{r}\right)$ satisfies

(i) $\left(A, i_{0}\right) \subset D\left((T R)^{r} T\right)$ and $(T R)^{r} T\left(A, i_{0}\right)=\left(A_{r},-i_{r}\right)$, then either

(a) $A_{r} \subset J_{-i_{r}} \cap(K-(V \cup W))$ or

(b) $\left(r+1, A_{r+1},-i_{r+1}\right)$ satisfies (i).

The proof of this given in [5] applies for our unbounded case. As in Lemma 7 [5], our lemma will be proved if we show that (i) cannot hold for all $r$. For then $k+1$ can be taken as the first $r$ for which (i) does not hold. So assume (i) holds for all $r$. Then $A_{r} \subset J_{-i_{r}}$, and $A_{r}$ is congruent to $A$, and hence an unbounded arc, for all $r \geqslant 0$. Therefore we have a sequence $\left(A_{0},-i_{0}\right),\left(A_{1},-i_{1}\right), \ldots$, where the terms of the sequence are disjoint by Lemma 4 [5]. Then there is a strictly increasing sequence $r_{1}, r_{2}, \ldots$ with $-i_{r_{j}}=i$ for some $i$ and all $j$, and $A_{r_{j}}$ $\subset J_{i}$ for $j=1,2, \ldots$ and the $A_{r_{j}}$ 's are disjoint and congruent. This is impossible since $D_{i}$ is a topological half-plane so any three unbounded subarcs of $J_{i}$ cannot be disjoint.

Corollary 6.2-1. For every $A^{i} \in U, p\left(A^{i}\right) \in U$.

Proof. See Lemma 8 [5]. 
Lemmas 9 [5]-13 [5] and the necessary definitions are valid for the unbounded arcs of $U$.

Definition [5]. $K_{+-}$is the set-theoretic union of all $A \in D(p)$ such that $A \subset K_{+}$and $p(A) \subset K_{-}$; similarly for $K_{-+}, K_{++}$and $K_{--}$.

For the remainder of this paper let us restrict $E_{+}$and $E_{-}$so that the arcs in $\mathrm{U}$ are convex.

THEOREM 6.3. If two eventually convex figures are scissor congruent their boundaries are eventually scissor congruent.

Proof. Suppose $A^{1} \in U \cap K_{++}$.

Case (a). $A^{1}$ is convex but not a ray. Let $E^{k}=T_{i_{k}} \cdots T_{i_{0}} E_{+}$, where $T_{i_{k}} \cdots T_{i_{0}}$ is the rigid motion described in Lemma 6.2. By Lemma 12 [5], $E^{k}$ and $E_{+}$are on opposite sides of $p\left(A^{1}\right)$, but $p\left(A^{1}\right)$ is part of the boundary of $E^{k}$ which is congurent to $E_{+}$. This is impossible if $A^{1}$ is convex and not a ray.

Case (b). $A^{1}$ is a ray and $A^{-1}, A^{-2} \in U \cap K_{-}$and are both convex but not rays. Then by Case (a) $A^{-1}, A^{-2} \in U \cap K_{-+}$. But $p\left(A^{-1}\right) \neq p\left(A^{-2}\right)$ since $p$ is 1-1. Impossible unless one of them, say $A^{-1}$, is a ray. Therefore $A^{1}$ is congruent to $A^{-1}$.

7. Invariance of angle under scissor congruence. In this section and the next we will use the following notation.

DEFinition 7.1. $E_{+}$and $E_{-}$are properly situated. For $j=1,2$ :

(i) For each $A^{j} \in U$, let $M^{j}=T_{i_{k}} \cdots T_{i_{0}}$ described in Lemma 6.2. Let us index the $A^{j}$ 's so that $M^{j}\left(A^{j}\right)=p\left(A^{j}\right)=A^{-j}$.

(ii) Let $a_{j}=\left(x_{j}, g_{+}\left(x_{j}\right)\right)$ be the endpoint of $A^{j}$, where $K_{+}$is the graph of $g_{+}$; and let $a_{-j}=\left(x_{-j}, g_{-}\left(x_{-j}\right)\right)$ be the endpoint of $A^{-j}$, where $K_{-}$is the graph of $g_{-}$.

We can assume that $x_{1}<x_{2}$ and $x_{-1}<x_{-2}$. Let $l_{1}\left(l_{2}\right)$ be the ray with endpoint $a_{1}\left(a_{2}\right)$ which is tangent to $A^{1}\left(A^{2}\right)$ at $a_{1}\left(a_{2}\right)$ from the left (right), with $l_{1} \cup l_{2} \cup \overline{a_{1} a_{2}}$ a wedge containing an unbounded subset of $E_{+}$. Let $l_{-j}=M^{j}\left(l_{j}\right)$.

THEOREM 7.2. The rigid motions $M^{1}$ and $M^{2}$ are translations.

Proof. Let $B_{j}=$ the topological half-plane bounded by $l_{j}$ and $A^{j}, j=1,2$, $-1,-2$. (If $A^{j}$ is a ray then let $B_{j}=\varnothing$.)

Then we have that $B_{-j}=M^{j}\left(B_{j}\right), j=1,2$. Therefore we have that $E_{+} \cup$ $B_{1} \cup B_{2}$ is scissor congruent to $E_{-} \cup B_{-1} \cup B_{-2}$ since

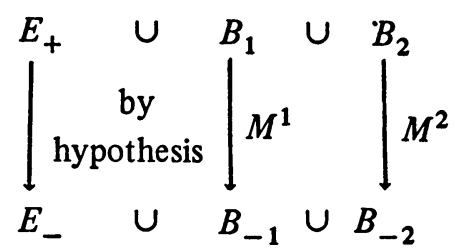


By Lemma 5.6, the two wedges $E_{+} \cup B_{1} \cup B_{2}$ and $E_{-} \cup B_{-1} \cup B_{-2}$ have equal angles, i.e. the angle, $\theta$, between $l_{1}$ and $l_{2}$ is the same as the angle between $l_{-1}$ and $l_{-2}$. Since $E_{+}$and $E_{-}$are properly situated and $A^{j} \cong A^{-j}, l_{j}$ is parallel to $l_{-j}, j=1,2$. Therefore $M^{1}$ and $M^{2}$ are translations.

COROLlaRY 7.2-1. Two eventually convex scissor congruent figures have the same angle.

Proof. Follows immediately from Lemma 7.2.

LEMMA 7.3. If an arc, $A$, is congruent to a proper subarc of itself by a plane rigid motion, the rigid motion is the product of a translation and reflections. If $A$ is convex, then it is a ray.

Proof. Let $M$ be a plane rigid motion such that $M(A) \cong$ proper subarc of $A$. Let $p_{0}$ be a point of $A$ such that $p_{0} \neq M\left(p_{0}\right)$. In general, let $p_{i}=M\left(p_{i-1}\right)$, $i=1,2, \ldots$ Let $P$ be the polygonal arc $\overline{p_{0} p_{1}}+\overline{p_{1} p_{2}}+\ldots$. If $A$ is convex it must lie on one side of $P$. If we show that $P$ is a ray, then a convex arc, $A$, must be a ray since it intersects $P$ infinitely often. Let $\theta_{i}=$ exterior angle at $p_{i}$, measured from $\overline{p_{i-1} p_{i}}$ (extended) to $\overline{p_{i} p_{i+1}} .0 \leqslant \theta_{1} \leqslant \pi$, and assume positive $\theta_{1}$ is measured clockwise.

If $\theta_{1}=0$, then our arc, $P$, is an infinite ray, and $M=T R$, where $T$ is a translation and $R$ is a reflection such that $R(P)=P$. Therefore assume $\theta_{1}>0$. See Figure 1 .

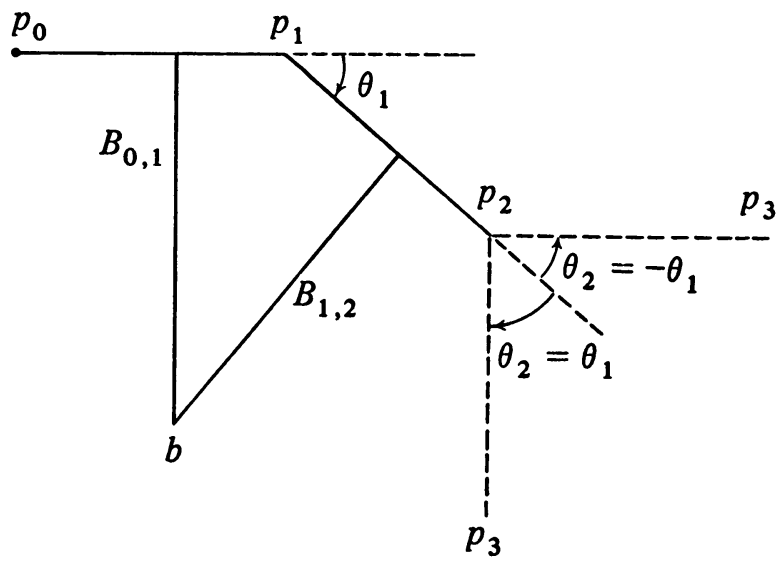

FIGURE 1

Since $M\left(\overline{p_{0} p_{1}}\right)=\overline{p_{1} p_{2}}, M$ is determined up to a reflection of the plane about $\overline{p_{1} p_{2}}$. (I.e. $M$ (plane) = plane, a plane is determined by three noncollinear points. Thus $p_{1}, p_{2}$ and $p_{3}$ determine $M$ (plane).) Therefore $\theta_{2}=-\theta_{1}$ or else $\theta_{1}$.

Case (a). If $\theta_{2}=\theta_{1}$ : Let $B_{i, i+1}=$ perpendicular bisector of $\overline{p_{i} p_{i+1}}, i=0$, $\ldots \ldots M\left(B_{i, i+1}\right)=B_{i+1, i+2}$. Let $b=B_{0,1} \cap B_{1,2} \neq \varnothing$ if $\overline{p_{0} p_{1}}$ and $\overline{p_{1} p_{2}}$ are not collinear (i.e. if $P$ is not an infinite ray). 
Therefore $b=B_{0,1} \cap M\left(B_{0,1}\right), M(b)=M\left(B_{0,1}\right) \cap M\left(B_{1,2}\right)$ and $b$ is equidistant from $p_{0}, p_{1}$ and $p_{2} . b$ is a fixed point. Thus $M$ reduces to a rotation about the point $b$ through an angle $\theta_{1}$. In general, for $i \geqslant 0, p_{i}, p_{i+1}$ are equidistant from $b$, length $\overline{p_{i} p_{i+1}}=$ length $\overline{p_{i+1} p_{i+2}}$, and $\theta_{i}=\theta_{i+1}=\theta_{1}$. For $\theta_{1}>0$, there exists a least $n$ such that $n \theta_{1} \geqslant 2 \pi$.

(i) If $n \theta_{1}=2 \pi$, then $P$ is the boundary of a regular $n$-gon, which is not congruent to a proper subarc of itself.

(ii) If $n \theta_{1}>2 \pi$, then since length $\overline{b p_{0}}=$ length $\overline{b p}, \overline{p_{n-1} p_{n}}$ must intersect $\overline{p_{0} p_{1}}$, i.e. $A$ would intersect itself and hence not be an arc.

Therefore $\theta_{1}=0$.

Case (b). If $\theta_{2}=-\theta_{1} \neq 0$. This is impossible if $A$ is convex. But if $A$ is not convex, then $\theta_{3}=-\theta_{2}=\theta_{1}$, etc. Thus $\overline{p_{i} p_{i+1}}$ and $\overline{p_{i+2} p_{i+3}}$ are parallel line segments, for $i=0,1, \ldots$, and $p_{0}, p_{2}, p_{4}, \ldots$ lie along a ray and $p_{1}, p_{3}$, ... all lie on the same side of this ray. Thus there is a translation, $T$, such that $T=M^{2}$. So if $M$ contains a rotation factor that is nontrivial, it must be a rotation of $\pi$ radians about some point $x$. Call the rotation $R_{x}$. Let $L_{1}$ and $L_{2}$ be any two perpendicular lines intersecting at $x$. Then $R_{x}$ is equivalent to a reflection about $L_{1}$ followed by a reflection about $L_{2}$. Therefore $M$ is the product of a translation and reflections.

THEOREM 7.4 (Classification of the mappings $M^{1}$ and $M^{2}$ ). If $K_{+}$and $K_{-}$ do not contain a ray, and there exist convex arcs $A_{*}^{1}, A_{*}^{2} \subset K_{+}$and $A_{*}^{-1}, A_{*}^{-2} \subset$ $K_{-}$and rigid motions $M_{*}^{j}: A_{*}^{j} \cong A_{*}^{-j}, j=1,2$, then either $M_{*}^{1}=M^{1}$ and $M_{*}^{2}=$ $M^{2}$ or else $M^{1}=M_{*}^{2}\left(M^{2}\right)^{-1} M_{*}^{1}$.

Proof. For definiteness, suppose $A_{*}^{1} \subset A^{1}$. Then $M^{1}\left(A_{*}^{1}\right) \subset A^{-1}$ and $M_{*}^{1}\left(A_{*}^{1}\right) \cong M^{1}\left(A_{*}^{1}\right)$.

(i) If $A^{-1} \subset A_{*}^{-1}$ or $A_{*}^{-1} \subset A^{-1}$ then $M_{*}^{1}=M^{1}$ since $A_{*}^{-1}$ is convex and not a ray and hence does not contain a proper subarc to which it is congruent, by Lemma 7.3. Similarly, $M_{*}^{2}=M^{2}$.

(ii) If $A^{-1} \subset A_{*}^{-2}$ or $A_{*}^{-2} \subset A^{-1}$ there is an arc, $A \subset A_{*}^{1}$ which is congruent to a subarc of $A_{*}^{j}$ and $A^{j}$ for all $j=1,2,-1,-2$, i.e. $M^{1}(A) \cong$ $M_{*}^{2}\left(M^{2}\right)^{-1} M_{*}^{1}(A)$. Again, by Lemma $7.3 M^{1}=M_{*}^{2}\left(M^{2}\right)^{-1} M_{*}^{1}$.

8. An invariant of scissor congruent uniaxial figures. In this section we will assume that $E_{+}$is uniaxial. By Corollary $7.2-1, E_{-}$is also uniaxial.

Definition 8.1. For $E$ a uniaxial convex figure, a curved rectangle of $E$ is a bounded, closed, convex subset of $E$ whose boundary consists of two parallel line segments which are perpendicular to the axes of $E$ and the subarcs of the boundary of $E$ joining the endpoints of the line segments. The height of a curved rectangle is the distance between the parallel line segments. If a curved rectangle 
is an ordinary parallelogram choose the distance between the segments perpendicular to the axes.

THEOREM 8.2. If $Y$ is a line parallel to an axis of $E_{+}, M^{1}(Y)=M^{2}(Y)$.

Proof. Define $I_{+} \subset E_{+}$to be a strip with $\partial I_{+}=\bigcup_{j=1,2}$ axis with end. point $a_{j} \cup \overline{a_{1} a_{2}}$.

Let $I_{-}$be similarly defined in $E_{-}$. Our theorem will be proved if we show that $I_{+}$and $I_{-}$have equal widths. Let $\left|I_{+}\right|=$width of $I_{+},\left|I_{-}\right|=$width of $I_{-}$.

Let $H$ be a closed upper half-plane with boundary $l$ such that: (i) $H \cap D_{i} \neq \varnothing$ for $m<i \leqslant n$, and (ii) $a_{1}$ and $a_{2}$ are not in $H$. Such an $H$ obviously exists.

Let $C_{+}$be a curved rectangle of $H \cap E_{+}$with the lower boundary line of $C_{+}$ equal to $l \cap E_{+}$. Let $L$ denote the height of $C_{+}$.

For $i=1, \ldots, m$ : Each $T_{i}$ is a translation. So with each $T_{i}$ we can associate two real numbers, $x_{i}$ and $y_{i}$, such that for any point $(x, y)$ of the plane, $T_{i}(x, y)=$ $\left(x+x_{i}, y+y_{i}\right)$. Let $\epsilon / 2=\max _{i} y_{i}-\min _{i} y_{i}$.

Then there is a curved rectangle, $C_{-}$of $E_{-}$contained in $\bigcup_{1 \leqslant i \leqslant m} T_{i}\left(C_{+} \cap D_{i}\right)$ of height $L-\epsilon$, provided $L>\epsilon$. In fact, there are such pairs, $C_{+}$and $C_{-}$, with height $L$ and $L-\epsilon$ respectively, as large as we wish. Let $R_{-1}$ and $R_{-2}$ be the two curved rectangles of $E_{-}$such that $R_{-1} \cup R_{-2} \cup\left(C_{-} \cap I_{-}\right)=C_{-}$.

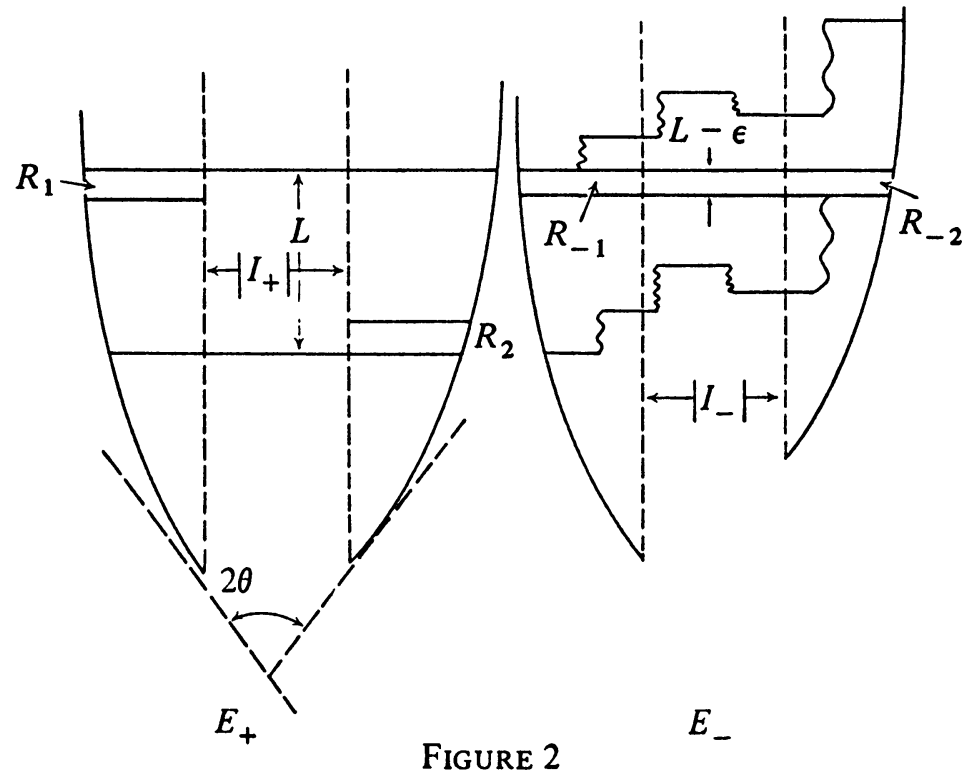

Since $E_{+}$and $E_{-}$are scissor congruent, their boundaries are eventually scissor congruent by Theorem 6.3. Thus there are two curved rectangles $R_{1}$ and $R_{2}$ which are contained in $C_{+}$and are congruent to $R_{-1}$ and $R_{-2}$, respectively.

Let $R_{+}=C_{+} \cap I_{+}$.

Let $S_{1}, \ldots, S_{4}$ be the four (possibly empty) curved rectangles of $C_{+}-$ 


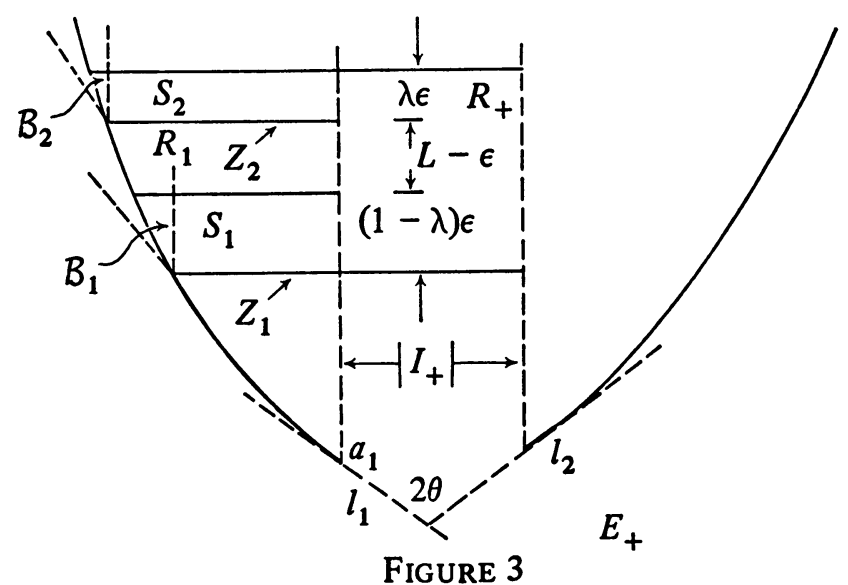

$\left(R_{+} \cup R_{1} \cup R_{2}\right)$. Refer to Figures 2 and 3 where some of the details are shown.

For $j=1, \ldots, 4:$ Let $Z_{j}=$ the length of the lower line segment of $\partial S_{j}$, and $s_{j}=$ the point of intersection of this segment with $K_{+}$. Let $K_{+}$be the graph of $g$. Let $F\left(s_{j}\right)=\min \left(\left|\left(g_{R}^{\prime}\left(s_{j}\right)\right)^{-1}\right|, \mid\left(g_{L}^{\prime}\left(s_{j}\right)\right)^{-1} i\right)$. (See page .) Let $B_{j}=$ $\left|\arctan F\left(s_{j}\right)\right|$. Let $2 \theta=$ angle between $l_{1}$ and $l_{2}$.

Let $Z=\Sigma_{j} Z_{j}$. We can choose $L \gg \epsilon$, in fact as large as we please. area $C_{+}$ $\geqslant$ area $C_{-}$. Therefore

$$
\sum_{j} \text { area } S_{j}+\operatorname{area}\left(R_{1} \cup R_{2}\right)+L\left|I_{+}\right|>(L-\epsilon)\left|I_{-}\right|+\operatorname{area}\left(R_{-1} \cup R_{-2}\right) \text {. }
$$

But $Z_{2} \leqslant L \tan B_{2}$ and $Z_{4} \leqslant L \tan B_{4}$, so

$$
\begin{aligned}
S_{1}+S_{2} & \leqslant \lambda \epsilon L \tan B_{2}+1 / 2(\lambda \epsilon)^{2} \tan B_{2}+1 / 2(1-\lambda)^{2} \epsilon^{2} \tan B_{1}+Z_{1}(1-\lambda) \epsilon \\
& \leqslant \epsilon L \tan B_{2}+1 / 2 \epsilon^{2} \tan B_{2}+\epsilon Z_{1}+1 / 2 \epsilon^{2} \tan \theta
\end{aligned}
$$

since $B_{1} \leqslant \theta$ because $E_{+}$is eventually convex and $a_{1}, a_{2} \notin$ int $H$, and $0 \leqslant \lambda \leqslant 1$. Similarly, $B_{3} \leqslant \theta$. Therefore,

$$
\begin{aligned}
\sum_{j} S_{j} \leqslant & \epsilon L\left(\tan B_{2}+\tan B_{4}\right)+\epsilon^{2}\left(\tan B_{2}+\tan B_{4}\right) \\
& +\epsilon\left(Z_{1}+Z_{2}\right)+\epsilon^{2} \tan \theta
\end{aligned}
$$

Therefore,

$$
\begin{aligned}
\epsilon\left(\epsilon \left(\tan B_{2}+\right.\right. & \left.\left.\tan B_{4}\right)+Z_{1}+Z_{2}+\epsilon \tan \theta+\left|I_{-}\right|\right) \\
& \geqslant L\left(\epsilon\left(\tan B_{2}+\tan B_{4}\right)+\left|I_{-}\right|-\left|I_{+}\right|\right) .
\end{aligned}
$$

But $B_{2} \rightarrow 0$ and $B_{4} \rightarrow 0$ since $E_{+}$is uniaxial and $L$ is unbounded. Thus $\left|I_{-}\right| \rightarrow$ $\left|I_{+}\right|$.

COROLLARY 8.2-1. Two strips which are scissor congruent have the same width.

Proof. Follows immediately from Theorem 8.2. 
9. Equivalence of scissor congruence and Sequivalence.

THEOREM 9.1. Two eventually convex figures are scissor congruent if and only if they are S-equivalent.

Proof. One way is Theorem 4.4. The other follows from Theorems 6.3, 7.2 and 8.2.

THEOREM 9.2. 'Is scissor congruent to' is an equivalence relation on the class of eventually convex figures.

Proof. Follows immediately from Theorem 9.1 and Lemma 3.11.

We can now prove Theorem 1.2, which we will restate.

THEOREM 1.2. Two unbounded convex figures, $E_{+}$and $E_{-}$, are scissor congruent if and only if the union of $E_{+}$and a finite number of topological discs is scissor congruent to the union of $E_{-}$and a finite number of topological discs.

Proof. Let $F_{+}=$the union of $E_{+}$and a bounded figure. Let $F_{-}=$the union of $E_{-}$and a (not necessarily the same) bounded figure. Then $E_{+}$is scissor congruent to $F_{+}$and $E_{-}$is scissor congruent to $F_{-}$by Theorem 1.1. Thus $F_{+}$ and $F_{-}$are scissor congruent if and only if $E_{+}$and $E_{-}$are since "is scissor congruent to' is a transitive relation by Theorem 9.2.

\section{REFERENCES}

1. F. Hausdorff, Grundzüge der Mengenlehre, Veit, Leipzig, 1914; photographic reproduction, Chelsea, New York, 1949. MR 11, 88.

2. S. Banach and A. Tarski, Sur la decomposition des ensembles de points en parties respectivement congruentes, Fund. Math. 6 (1924), 244-277.

3. L. A. Ljusternik, Convex figures and polyhedra, GITTL, Moscow, 1956; English transl., Dover, New York, 1963. MR 19, 57; 28 \#4427.

4.. V. G. Boltjanskii, Equivalent and equidecomposable figures, GITTL, Moscow, 1956; 1956; English transl., Heath, Lexington, Mass., 1963.

5. Lester Dubins, Morris Hirsch and Jack Karush, Scissor congruence, Israel J. Math. 1, 1963, 239-247. MR 29 \#2706.

6. Branko Grünbaum, Convex polytopes, Pure and Appl. Math., vol. 16, Interscience, New York, 1967. MR 37 \#2085.

7. L. Fejes Tóth, Lagerungen in der Ebene auf der Kugel und im Raum, Die Grundlehren der math. Wissenschaften, Band 65, Springer-Verlag, Berlin, 1953. MR 15, 248. \#6194.

8. H. W. Guggenheimer, Differential geometry, McGraw-Hill, New York, 1963. MR 27

9. John G. Hocking and Gail S. Young, Topology, Addison-Wesley, Reading, Mass., 1961. MR 23 \#A2857.

10. M. H. A. Newman, Elements of the topology of plane sets of points, 2 nd ed., Cambridge Univ. Press, Cambridge, 1951. MR 13, 483.

DEPARTMENT OF MATHEMATICS, UNIVERSITY OF CALIFORNIA, BERKELEY, CALIFORNIA 94720

Current address: Lawrence Livermore Laboratory, University of California, Livermore, California 94550 